\title{
Poderosa no Parto: demonstração do protótipo de aplicativo para o pré-natal
}

\author{
Luiz Jr da Silva Lopes ${ }^{1}$, Emilly G. M. da Silva ${ }^{1}$, Neusa Andrade ${ }^{2}$, Claudia M. \\ Messias ${ }^{3}$, Isabel C. F. da Cruz ${ }^{3}$ \\ ${ }^{1}$ Universidade Federal do Rio de Janeiro (UFRJ) \\ Rio de Janeiro - RJ - Brasil \\ ${ }^{2}$ Universidade Paulista (UNIP) \\ São Paulo - SP - Brasil \\ ${ }^{3}$ Universidade Federal Fluminense (UFF) \\ Niterói - RJ - Brasil \\ \{luizjunior.lopes, neusagenciaweb2, marimessi1512\}@gmail.com, \\ emilly.marques@labnet.nce.ufrj.br, isabelcruz@id.uff.br
}

\begin{abstract}
Due to the risk of infection by the crisis in the health sector due to the Covid-19 pandemic, pregnant women have restricted prenatal care and no face-to-face access to care about the psychoprophylactic method for coping with discomfort during labor. The objective of this work is to demonstrate the prototype of the application "Poderosa no Parto", which seeks to promote the teaching-learning-evaluation of the psychoprophylactic method to help the pregnant woman in the preparation for active and natural childbirth.
\end{abstract}

Resumo. Em razão do risco de infecção pela crise no setor de saúde devido à pandemia de Covid-19, a pessoa gestante tem o cuidado pré-natal restrito e sem acesso presencial ao cuidado sobre o método psicoprofilático para o enfrentamento do desconforto durante o trabalho de parto. $O$ objetivo deste trabalho é demonstrar o protótipo do aplicativo "Poderosa no Parto", que busca promover o ensino-aprendizagem-avaliação do método psicoprofilático para ajudar a pessoa gestante na preparação para o parto ativo e natural.

\section{Introdução}

Em um contexto de pandemia e crise no setor de saúde, a pessoa gestante tem o cuidado pré-natal restrito, sem o ensino presencial sobre o método psicoprofilático para o enfrentamento do desconforto durante o trabalho de parto ${ }^{1}$. Para o enfrentamento deste desafio, gestante e enfermeira(o) podem utilizar tecnologias digitais, tal como o aplicativo proposto, como um recurso facilitador do ensino-aprendizagem-avaliação no preparo para o parto. Além disso, mesmo em um contexto de profundas desigualdades sociais, a utilização de tecnologias móveis pela população brasileira é crescente, se tornando um recurso cada vez mais apropriado pela enfermagem para diversos propósitos [Lima \& Barbosa 2019]. O objetivo deste trabalho é apresentar o protótipo de uma aplicação, para uso em desktops e smartphones, sobre o método psicoprofilático de preparo para o parto, visando o seu aprimoramento a partir das apreciações.

\footnotetext{
${ }^{1}$ Cruz, I. C. F. da; Silva, E. G. M. da; Siva Lopes, L. J. da - App Preparo para o Parto Natural. Hackcovid19, FIOCRUZ, RJ, 2020. Disponível em: http://www.cbpf.br/hackcovid19/Hackcovid_Desafios_Popula\%C3\%A7\%C3\%B5es_vulner\%C3\%A1veis .html
} 


\section{Método}

Trata-se de uma produção tecnológica do tipo prototipagem que visa à construção de uma aplicação Web/Mobile para auxiliar a pessoa gestante no pré-natal. O estudo foi baseado no Design Instrucional Sistemático (DIS), sendo adotado o modelo ADDIE (Analyze, Design, Develop, Implement, Evaluate) [Falcade et al. 2016]. No entanto, o protótipo foi desenvolvido em três etapas: Analyze, Design, Develop ${ }^{2}$. As ferramentas adotadas no desenvolvimento Web foram: React, Node.js, MongoDB e GitHub. Para fins de desenvolvimento, a plataforma Heroku foi escolhida para hospedagem.

\section{Resultados}

O conteúdo pedagógico do aplicativo baseou-se no curso "Preparo para o Parto e Aleitamento". O protótipo apresenta instrumentos para lembrar a gestante diariamente das técnicas orientadas pela(o) enfermeira(o), assim como recursos para facilitar a realização correta das técnicas, conscientização sobre o trabalho de parto e controle do desempenho em relação às atividades propostas para o aprendizado. O aplicativo recebeu o nome "Poderosa no Parto", e pode ser vizualizado através do endereço (https://poderosanoparto-frontend.herokuapp.com/), seus recursos são: perfil da usuária, agenda de tarefas com foco em treinamento da respiração superficial e profunda, relaxamento muscular progressivo e gráficos sobre o progresso nas atividades, incluindo um manual sobre práticas saudáveis para o parto, baseado em evidências científicas ${ }^{3}$.

\section{Conclusão}

O uso de aplicativos em saúde é uma ferramenta tecnológica com potencial para melhorar o acompanhamento da pessoa gestante durante o pré-natal. $\mathrm{O}$ desenvolvimento do protótipo por uma equipe composta por profissionais de saúde e TI é um diferencial positivo desta experiência. No momento deste estudo, está sendo analisada a forma de adaptação da versão Web para a versão Mobile.

\section{References}

Lima, C. S. P., \& Barbosa, S. D. F. F. (2019). Aplicativos móveis em saúde: caracterização da produção científica da enfermagem brasileira. Revista Eletrônica de Enfermagem, 21.

Falcade, A., Krassmann, A., Freitas, V., Kautzmann, T., \& Medina, R. D. (2016). Design Instrucional: um comparativo de metodologias para definição de abordagem em mundo virtual. In Brazilian Symposium on Computers in Education (Simpósio Brasileiro de Informática na Educação-SBIE) (Vol. 27, No. 1, p. 80).

\footnotetext{
${ }^{2}$ Lopes, L. J. S.; Silva, E. G. M.; Andrade, N. M. de; Messias, C. M.; Cruz, I. C. F. da. Processo de desenvolvimento de um aplicativo de educação em saúde para a pessoa gestante. Jornada Acadêmica de Educação em Saúde Digital - JAESD, Universidade Federal do Ceará, CE, 2020. Disponível em: http://www.nuteds.ufc.br/1a-jornada-academica-de-educacao-em-saude-digital-jaesd/

${ }^{3}$ Cruz, I. C. F. da - Preparo para o Parto e Aleitamento. Niterói, RJ, 2020. [Acesso em 25 de maio de 2021]. Disponível em: http:www.nepae.uff.br/
} 